\title{
Euler Graph Research Based on Incidence Matrix Main Diagonal Spectrum Theory
}

\author{
WANG Xiao-ping ${ }^{1}$, ZHAO Kai $^{2 *}$ \\ 1. Humanities and Social Science Department of Yibin Vocational and Technical College, Yibin, \\ 644003 \\ 2. Modern Manufacturing Engineering Department of Yibin Vocational and Technical College, Yibin, \\ 644003 \\ *Corresponding author:kijidi@sina.com
}

Keywords:graph theory; incidence matrix; main diagonal spectrum; Seven Bridges of Königsberg

\begin{abstract}
By using the vertex - edge incidence matrix, the vertex incidence matrix and edge incidence matrix was established,also using the incidence matrix main diagonal spectrum to prove Euler graph problem in the paper.
\end{abstract}

\section{Introduction}

This paper finds that there are some problems about the theory of incidence matrix and adjacency matrix in current matrix theory and graph theory. There are no inference of the relationship between incidence matrix and adjacency matrix in the current literature of graph theory [1-7]. These two matrixes are artificially set separately and the value of the main diagonal of the adjacency matrix is also artificially set, this could result in the loss of important information; graph theory could be improved by introducing matrix, the relationship between graph theory and matrix theory is close, the application of matrix theory in graph theory could take the benefit of computing capacity of computer for matrix, matrix theory can improve the development of graph theory. This paper studies the matrix theory in graph theory. The following firstly introduce a few essential definitions and attributes between graph theory and matrix theory.

\section{Definition of Incidence Matrix}

Definition 1: For a connected graph $G=\langle V, E\rangle$ with vertex set of $V=\left\{v_{1}, v_{2}, \cdots, v_{m}\right\}$ and edge set of $E=\left\{e_{1}, e_{2}, \cdots, e_{n}\right\}$, its vertex-edge incidence matrix is $K(G)=\left(k_{i j}\right)_{m \times n}$, where $k_{i j}=\left\{\begin{array}{c}1, \quad \text { whenv }_{i} \text { ande }_{j} \text { arerelated, } \\ 0, \text { when }_{i} \text { ande }_{j} \text { arenotrelated. }\end{array}\right.$ Matrix $K(G)$ is also known as the characteristic matrix of graph $G$. The transpose of $K(G)$ is denoted by $K^{T}(G)$.

Definition 2: The vertex incidence matrix of graph $G$ is $V(G)=\left(v_{i j}\right)_{m \times m}$, and $V(G)=K(G) K^{T}(G)$. Obviously, $V(G)$ is a symmetric matrix.

Definition 3: The edge incidence matrix of graph $G$ is $E(G)=\left(e_{i j}\right)_{n \times n}$, and $E(G)=K^{T}(G) K(G), E(G)$ is a symmetric matrix.

Attribute 1: the vertex incidence matrix $V(G)=\left(v_{i j}\right)_{m \times m}$, when $i=j$ which is $v_{i i}=\sum_{i=1}^{m} v_{i j}(i \neq j)$ or $v_{i i}=\sum_{j=1}^{m} v_{i j}(i \neq j)$, the value of $v_{i i}$ is the sum of connections between vertexes and edges.

Attribute 2: the edge incidence matrix $E(G)=\left(e_{i j}\right)_{n \times n}$, when $e_{i i}=0$, this matrix becomes the adjacency matrix of the graph theory. 
Attribute 3: the traces of the vertex incidence matrix and the edge incidence matrix are equal, which is $\operatorname{tr}(V)=\operatorname{tr}(E)$.

Definition 4: The main diagonal spectrum of the vertex incidence matrix $V(G)$ of graph $G$ is $\operatorname{Diag}-\operatorname{Spec} V(G)$, set

$$
\operatorname{Diag}-\operatorname{Spec} V(G)=\left[\begin{array}{llll}
d_{v 1} & d_{v 2} & \cdots & d_{v s} \\
z_{v 1} & z_{v 2} & \cdots & z_{v s}
\end{array}\right]
$$

Where, $d_{v i}$ is the $i^{\text {th }}$ different value in the main diagonal of the square matrix $V(G)$ of order $m$. the value increases and the degree of the vertex is $z_{v i}$, which means $s \leq m$ that the number of different values cannot be greater than the number of vertexes. When all the values on the main diagonal are different, $s=m ; z_{v i}$ is the number of elements that equal $d_{v i}$ on the main diagonal, which is called the multiplicity of the vertex with the degree of $d_{v i}$, means there are $z_{v i}$ vertexes with the degree of $d_{v i}, m=\sum_{j=1}^{s} z_{v j}$. Actually, the main diagonal spectrum of a matrix is the statistic of the values on the main diagonal of the square matrix.

Attribute 4: the relationship between the trace of the vertex incidence matrix $\operatorname{tr}(V)$ and $\operatorname{Diag}-\operatorname{Spec} V(G)$ is $\operatorname{tr}(V)=\sum_{i=1}^{s} d_{v i} z_{v i}$.

Definition 5: The main diagonal spectrum of the edge incidence matrix $E(G)$ of graph $G$ is $\operatorname{Diag}-\operatorname{SpecE}(G)$, set

$$
\operatorname{Diag}-\operatorname{SpecE}(G)=\left[\begin{array}{llll}
d_{e 1} & d_{e 2} & \cdots & d_{e s} \\
Z_{e 1} & Z_{e 2} & \cdots & z_{e s}
\end{array}\right]
$$

$\operatorname{Diag}-\operatorname{Spec} E(G)$ is called the main diagonal spectrum of the edge incidence matrix $E(G)$, it is the same with the definition of the main diagonal spectrum of the edge incidence matrix.

\section{The incidence matrix of the Seven Bridges of Königsberg}

The Seven Bridges of Königsberg was a historically notable problem in mathematics. It has been solved by Leonhard Euler by graph theory. The conclusion is that there is no solution to find a walk through the city that would cross each bridge once and only once. Euler laid the foundations of a new discipline based on this problem, which is graph theory. Let us start with the problem, Figure 1 shows the layout of the seven bridges and the connected graph $G$. Based on the vertexes $\left(v_{1}, v_{2}, v_{3}, v_{4}\right)$ and the edges $\left(e_{1}, e_{2}, e_{3}, e_{4}, e_{5}, e_{6}, e_{7}\right)$, the vertex and edge incidence matrix is $Z(G)$ and the vertex incidence matrix $V(G)=K(G) K^{T}(G)$ and the edge incidence matrix is $E(G)=K^{T}(G) K(G)$.
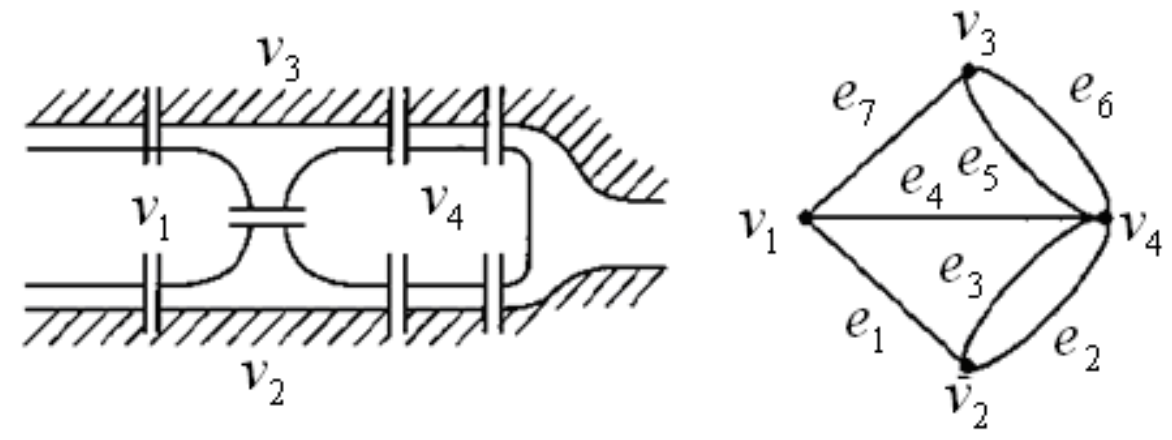

Figure 1. The layout of the seven bridges and the connected graph 


$$
\begin{aligned}
& K(G)=\left[\begin{array}{lllllll}
1 & 0 & 0 & 1 & 0 & 0 & 1 \\
1 & 1 & 1 & 0 & 0 & 0 & 0 \\
0 & 0 & 0 & 0 & 1 & 1 & 1 \\
0 & 1 & 1 & 1 & 1 & 1 & 0
\end{array}\right] \quad K^{T}(G)=\left[\begin{array}{llll}
1 & 1 & 0 & 0 \\
0 & 1 & 0 & 1 \\
0 & 1 & 0 & 1 \\
1 & 0 & 0 & 1 \\
0 & 0 & 1 & 1 \\
0 & 0 & 1 & 1 \\
1 & 0 & 1 & 0
\end{array}\right] \\
& V(G)=K(G) K^{T}(G)=\left[\begin{array}{llll}
3 & 1 & 1 & 1 \\
1 & 3 & 0 & 2 \\
1 & 0 & 3 & 2 \\
1 & 2 & 2 & 5
\end{array}\right] \quad E(G)=K^{T}(G) K(G)=\left[\begin{array}{lllllll}
2 & 1 & 1 & 1 & 0 & 0 & 1 \\
1 & 2 & 2 & 1 & 1 & 1 & 0 \\
1 & 2 & 2 & 1 & 1 & 1 & 0 \\
1 & 1 & 1 & 2 & 1 & 1 & 1 \\
0 & 1 & 1 & 1 & 2 & 2 & 1 \\
0 & 1 & 1 & 1 & 2 & 2 & 1 \\
1 & 0 & 0 & 1 & 1 & 1 & 2
\end{array}\right]
\end{aligned}
$$

$$
\operatorname{Diag}-\operatorname{Spec} V(G)=\left[\begin{array}{ll}
3 & 5 \\
3 & 1
\end{array}\right] \operatorname{Diag}-\operatorname{Spec} E(G)=\left[\begin{array}{l}
2 \\
7
\end{array}\right]
$$

In the main diagonal spectrum of the edge incidence matrix $\operatorname{Diag}-\operatorname{Spec} E(G)=\left[\begin{array}{l}2 \\ 7\end{array}\right], 7$ is the number of edges, and 2 means each edge has two vertexes. According to Attribute 4, the trace of the edge incidence matrix $\operatorname{tr}(E)=14$. According to Attribute 3, the trace of the vertex incidence matrix $\operatorname{tr}(V)=14$. The values of the traces of the vertex and edge incidence matrixes must be even, which means there is no odd number of odd vertexes in the connected graph.

Based on this point, vertexes $K(G) 、 V(G)$ and $E(G)$ are connected, which means once one vertex is known, the other two matrixes can be derived. For normal connected graphs, the number of vertexes is less than the number of edges. So the data of the vertex incidence matrix is the least, but the other two matrixes can be derived based on the vertex incidence matrix. The vertex incidence matrix is a symmetric matrix. Additionally, according to Attribute 3, the elements on the main diagonal can be derived by the row or column of the adjacency matrix. So the actual data that need to be stored is just $\frac{m^{2}-m}{2}$. In sum, the vertex incidence matrix can provide the same information by the least data.

\section{The demonstration of Euler graph by the main diagonal spectrum}

According to the Eulerian path:

(1) All connected graphs with all vertices of even degree have an Eulerian circuit or Eulerian cycle. An Euler path is a trail in a graph which visits every edge exactly once.

(2) All the connected graphs with exactly two vertices of odd degree have an Eulerian circuit or Eulerian cycle, all Eulerian trails start at one of them and end at the other. They are called semi-Eulerian.

(3) Other graphs are not Euler graph.

According to the vertex main diagonal spectrum $\operatorname{Diag}-\operatorname{Spec} V(G)=\left[\begin{array}{llll}d_{v 1} & d_{v 2} & \cdots & d_{v s} \\ z_{v 1} & z_{v 2} & \cdots & z_{v s}\end{array}\right]$, the following is the workflow to find a Euler graph: 


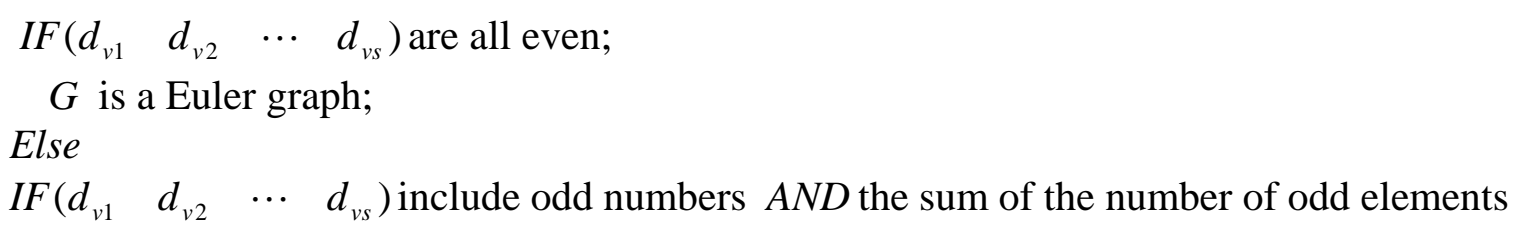
in the columns of $\left(\begin{array}{lllll}d_{v 1} & d_{v 2} & \cdots & d_{v s}\end{array}\right)$ and $\left(\begin{array}{llll}z_{v 1} & z_{v 2} & \cdots & z_{v s}\end{array}\right)$ is 2;

\section{Else}

$G$ is semi-Eulerian;

END

$G$ is not Euler graph;

The demonstration of the Seven Bridges of Königsberg by the main diagonal spectrum:

Determining Euler graph by the main diagonal spectrum of the vertex incidence matrix, the main diagonal spectrum of the graph of the Seven Bridges of Königsberg is $\operatorname{Diag}-\operatorname{Spec} V(G)=\left[\begin{array}{ll}3 & 5 \\ 3 & 1\end{array}\right]$, the values of the main diagonal are 3 and 5, which are odd, and all of the four vertexes are odd. That means there are seven odd vertexes in the connected graph of the Seven Bridges of Königsberg instead of two. According to the previous workflow, this graph is not Euler graph and there is no solution for the Seven Bridges of Königsberg.

\section{Conclusions}

This paper finds the connections between graph theory and matrix theory which have not been found in current literature. This paper derives the vertex incidence matrix and the edge incidence matrix through the adjacency matrix of vertexes and edges, defines the main diagonal spectrum of square matrixes, explains the meaning of the main diagonal spectrum and traces in graph theory, and determine graphs are Euler graph, semi-Eulerian or none. These incident matrixes can be used in other disciplines like mechanism rather than just graph theory.

\section{References}

[1] Zhang, X. D., Z. L. Li. Graph Theory and Its Applications. Beijing, China Higher Education Press, 2005.

[2] Wang, C. R. Graph Theory, $3^{\text {rd }}$ edition. Beijing, Beijing Institute of Technology Press, 2001.

[3] Xu, J. M. Graph Theory and Its Applications, $3^{\text {rd }}$ edition. Anhui: University of Science and Technology of China Press, 2010.

[4] James E. Gentle. Matrix Algebra Theory, Computations, and Applications in Statistics [M] Springer Science \& Business Media,2007.

[5] Handbook of Graph Theory (SECOND EDITION) [C]. CRC Press Taylor \& Francis Group.2014

[6] J.A. Bondy, U.S.R. Murty. Graph Theory [M].Springer. 2008.

[7] Alan Jeffrey. Matrix Operations for Engineers and Scientists [M] Springer Science \& Business Media B.V. 2010 